\title{
Frecuencia de Hueso Lambdoideo en Cráneos con Deformación Artificial en el Norte de Chile
}

\author{
Frecuency of Wormian Lambdoid Bone in Skulls with Artificial Deformation in Northern Chile
}

"Fernando García-Hernández \& ** Gabriel Murphy-Echeverría

GARCÍA-HERNÁNDEZ, F. \& MURPHY-ECHEVERRÍA, G. Frecuencia de hueso lambdoideo en cráneos con deformación artificial en el norte de Chile. Int. J. Morphol., 27(3):933-938, 2009.

RESUMEN: Los huesos suturales o wormianos son huesos accesorios, de importancia genética y hereditaria, considerados variables étnicas (Orts Llorca, 1958; Figún \& Garino, 1992). Se caracterizan por ser diversos en número, tamaño y forma, ubicados en suturas y fontículos, de preferencia en suturas posteriores, predominando la frecuencia a nivel de sutura lambdoidea o parieto-occipital (Sappey 1888, Sicher, 1965; Sánchez-Lara et al., 2004). Son de interés para la anatomía humana, antropología física, imagenología y medicina legal (Lips \& García-Hernández, 1983; Henríquez-Pino et al., 1992; Braga et al., 2000; García-Hernández et al., 2007). La deformación craneal, patológica o artificial, estimula la formación de centros accidentales de osificación, incrementando la presencia de osículos en suturas y fontículos (Del Papa \& Pérez, 2007). La deformación craneal artificial era común en diversas culturas amerindias, con propósito estético, mágico, religioso, como forma de identificación étnica, social, nobleza o asociada al grupo gobernante (TorresRouff, 2007) y se realizaba aplicando tablas de madera o vendajes compresivos en la cabeza del recién nacido. La hipótesis de investigación es determinar si existe mayor número de osículos lambdoideos en cráneos de atacameños del norte de Chile con y sin deformación craneal artificial. Para cumplir este objetivo se estudiaron 77 cráneos atacameños de ambos sexos (27 deformados y 50 no deformados), seleccionados al azar entre 293 individuos exhumados del cementerio Coyo-Oriente, datados entre 300-1200 D.C. y que forman parte del Museo R.P. Gustavo Le Paige en San Pedro de Atacama de la Universidad Católica del Norte. Los cráneos fueron analizados, fotografiados y mensurados para determinar el índice craneano. Existe prevalencia de huesos lambdoideos en cráneos deformados $(\mathrm{p}<0,05=0,00000249247)$, con promedio de indice craneal con mayor hiperbraquicefalia $(92,63$ DS 9,72$)$ y porcentaje de cráneos braquicefálicos con huesos lambdoideos $(92,6 \%-32 \%$; $<<0,05=3,67073 \mathrm{E}-7)$.

PALABRAS CLAVE: Braquicefalia; Hueso lambdoideo; Deformación craneana; Norte de Chile.

\section{INTRODUCCIÓN}

Los huesos suturales o wormianos son huesos accesorios, de importancia genética y hereditaria, considerados como variables étnicas (Orts Llorca, 1958; Figún \& Garino, 1992). Se caracterizan por ser diversos en número, tamaño y forma, ubicados en suturas y fontículos, de preferencia en suturas posteriores, predominando la frecuencia a nivel de sutura lambdoidea o parieto-occipital (Sappey 1888, Sicher, 1965; Sánchez-Lara et al., 2004). Son de interés para la anatomía humana, antropología física, imagenología y medicina legal (Lips \& García-Hernández, 1983; Henríquez-Pino et al., 1992; Braga et al., 2000; García-Hernández et al., 2007).

Los huesos suturales, son también conocidos como huesos wormianos, llamados así gracias a la completa des- cripción que hizo en 1611, el anatomista danés Olaüs Wormius o Worm (Sappey; Testut \& Latarjet, 1959). Se desarrollan por modificaciones en la formación de huesos anchos del cráneo a causa de alteraciones metabólicas del mesodermo, formándose a partir del tejido conectivo (Kahle et al., 1977), por centros de osificación anómalos (Orts Llorca), existiendo cierta correlación con la asimetría craneal, malformaciones de los huesos occipital y esfenoides, metopismo y anomalías congénitas del sistema nervioso central (Henríquez-Pino et al.; Braga et al.; García-Hernández et al.).

La deformación craneal, patológica o artificial, estimula la formación de centros accidentales de osificación, incrementando la formación de huesos wormianos en suturas

\footnotetext{
* Unidad de Anatomía, Departamento Biomédico, Facultad de Ciencias de la Salud, Universidad de Antofagasta, Antofagasta, Chile.

${ }^{* *}$ Cirujano Dentista, Antofagasta, Chile.
} 
y fontículos y están particularmente influenciados por todo lo relacionado con dichas prácticas de deformación craneal (Ossenberg, 1970; Pucciarelli, 1974; Barnes, 1994, citados por Del Papa \& Pérez, 2007).

La práctica de la deformación craneal se realizaba comúnmente en las diversas culturas amerindias y en forma particular en las regiones andinas. Se efectuaba mediante la aplicación de tablas de madera o vendajes compresivos en la cabeza del individuo recién nacido para modificar el eje de crecimiento aprovechando la maleabilidad de los huesos del cráneo. Tenía un propósito estético, mágico, religioso, como método de identificación étnica, social, nobleza o asociada al grupo gobernante (Meskell, 1998; Carod-Artal \& Vásquez-Cabrera, 2004; Shijman, 2005; Torres-Rouff, 2007).

La deformación tabular, realizada mediante tablas de madera, altera el eje de crecimiento craneal en sentido apical, formando cráneos braquicefálicos, mientras que la deformación anular o circular, usando vendajes compresivos, establece un eje longitudinal de crecimiento, formando cráneos con bóveda craneal dolicocéfala (Torres-Rouff).

Los huesos wormianos o suturales es el rasgo que más comúnmente aparece en las suturas de la bóveda craneal influenciado por la deformación craneal. En fuertes deformaciones craneales se ven afectada la frecuencia de los huesos suturales (Del Papa \& Pérez). En 1977, El-Najjar \& Dawson, señalaban que en cráneos fetales ya es detectable la presencia de huesos wormianos, restando importancia a la deformación craneal de tipo cultural como factor relevante en la formación de estos huesos. Reiteraban que los huesos wormianos o suturales se forman tanto en cráneos deformados como no deformados, sin diferencias significativas.

Sin embargo, investigaciones actuales reiteran la importancia de la deformación craneal en la formación de huesos suturales. En dicho sentido, se dirige lo investigado por O'Loughlin, 2004, quien señala que todo tipo de deformación craneal, ya sea por razones culturales o como resultado de craneosinostosis, afecta la frecuencia de cierto tipo de huesos wormianos o suturales. En particular, la deformación craneal aumenta la frecuencia significativa del osículo lambdoideo, aunque también aumenta la frecuencia de los osículos apical, parieto-mastoideo y occipito-mastoideo en algunos grupos de cráneos deformados culturalmente. Además, aunque varíen los grados o tipos de deformación craneal, todos los cráneos tienen mayor número de osículos lambdoideos que los cráneos no deformados.

Como señalan Carod-Artal \& Vásquez-Cabrera, la consecuencia inmediata de la deformación craneal es una alteración en el proceso normal de cierre de las suturas craneales, existiendo una correlación significativa entre la presencia de huesos wormianos posteriores y laterales en función de la deformación intencional. Todo parece indicar que el desarrollo de huesos wormianos en las suturas de la parte posterior se ven más afectadas por las fuerzas que las rodean en la deformación craneal artificial, que por lo que sucede en su contraparte anterior (O'Loughlin).

Los huesos wormianos son particularmente influenciados por todo lo que rodea al proceso de desarrollo postnatal y por ello no tiene relevancia el estudio respecto de la evolución asociada al parentesco. Pese a lo anterior, es interesante destacar que el parentesco evolutivo basado en la frecuencia de rasgos craneales no mensurables, puede ser más afectada en áreas geográficas donde las poblaciones practican deformación craneal artificial (Del Papa \& Pérez).

Los huesos suturales aparecen con mayor frecuencia en desórdenes en que se reduce o altera la osificación craneal, resultando deformaciones braquicefálicas (SánchezLara et al.). Sánchez-Lara et al., señalan que el incremento del número de huesos supernumerarios craneales está asociado a una mayor braquicefalización. Analizando diferentes índices craneanos de tipo braquicefálico en cráneos con deformación craneal no observan correlación estadística significativa con el número de huesos wormianos, encontrándose sí un aumento del número de huesos wormianos a medida que se incrementa la braquicefalia.

Por todos los antecedentes anteriores, nos planteamos la siguiente hipótesis: ¿Existe número mayor de huesos lambdoideos en cráneos braquicéfalos de atacameños con deformación craneal artificial respecto de cráneos no deformados del mismo grupo étnico?.

\section{MATERIAL Y MÉTODO}

La muestra consistió en 77 cráneos de atacameños prehispánicos de ambos sexos, del período post-Tiwanaku, que fueron elegidos al azar de un total de 293 individuos exhumados del cementerio Coyo-Oriente, datados entre 3001200 D.C. y que forman parte del Museo R.P. Gustavo Le Paige en San Pedro de Atacama, dependiente del Instituto de Investigaciones Arqueológicas de la Universidad Católica del Norte. A los restos óseos no se les realizó ningún tipo de intervención o consolidación por ser patrimonio cultural de la nación, estando solo autorizado el análisis y la observación de los cráneos, razón por lo cual fueron íntegramente fotografiados en todas sus normas anatómicas. También se mensuraron la longitud anteroposterior máxima del crá- 
neo y la anchura máxima de cráneo con la finalidad de determinar el índice craneano o craneal. La clasificación del índice craneal se detalla en Tabla I.

Los datos obtenidos fueron vertidos a una planilla electrónica de Microsoft Excel para su tabulación y para el estudio estadístico se utilizó el programa Statgraphics Plus 5.1

Tabla I. Clasificación de índice craneano o craneal (según R. Martin).

\begin{tabular}{lc}
\hline Tipo de bóveda craneal en norma & Rango de índice \\
\hline Cráneos Hiperdolicocéfalos & X a 69,9 \\
Cráneos Dolicocéfalos & 70,0 a 74,9 \\
Cráneos Mesocéfalos & 75,0 a 79,9 \\
Cráneos Braquicéfalos & 80,0 a 84,9 \\
Cráneos Hiperbraquicéfalos & 85,0 a X \\
\hline
\end{tabular}

\section{RESULTADOS}

Los resultados de los análisis estadísticos obtenidos en relación a cráneos atacameños deformados artificialmente y no deformados se muestran en las Tablas II y III y en Fig. 1 a 4.

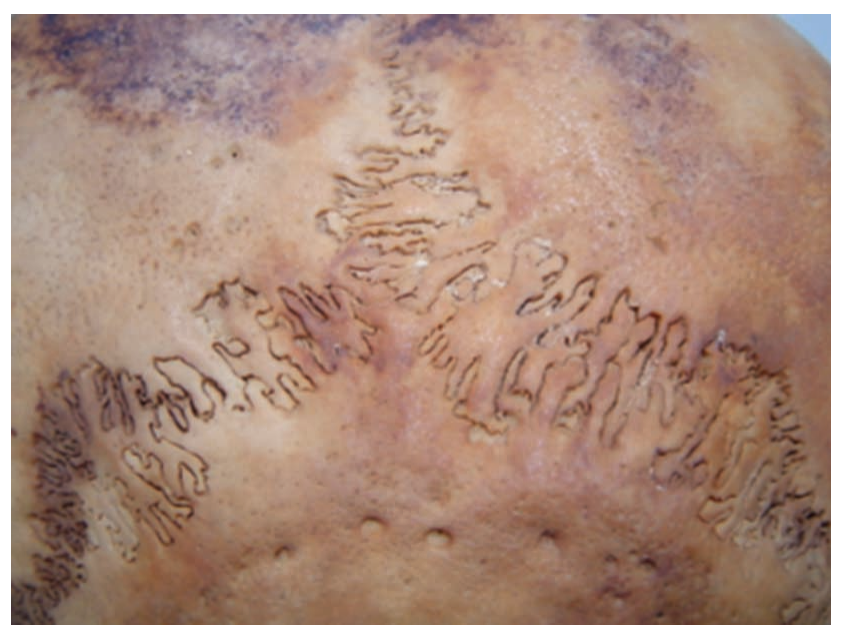

Fig. 1. Huesos lambdoideos bilaterales en cráneo deformado artificialmente.

Tabla II. Características morfológicas de cráneos atacameños ( $\mathrm{n}=77) .1$ p <0,05=0,0000424296 con diferencia estadística significativa al $95 \%$ entre promedios de longitud anteroposterior en cráneos deformados y no deformados. 2 p $<0,05=0,0313775$ con diferencia estadística significativa al 95\% entre promedios de anchura máxima en cráneos deformados y no deformados. 3 p- $<0,05=0,0000266496$ con diferencia estadística significativa al $95 \%$ entre promedios de índice craneano en cráneos deformados y no deformados.

\begin{tabular}{|c|c|c|c|c|c|c|c|c|c|}
\hline \multirow[t]{3}{*}{ Tipo de cráneo } & \multirow{2}{*}{\multicolumn{2}{|c|}{$\begin{array}{c}\text { Longitud } \\
\text { anteroposterior del } \\
\text { cráneo }\end{array}$}} & \multirow{2}{*}{\multicolumn{2}{|c|}{$\begin{array}{c}\text { Anchura máxima } \\
\text { del cráneo }\end{array}$}} & \multicolumn{2}{|c|}{ Indice craneano } & \multicolumn{3}{|c|}{ Cráneos según forma bóveda } \\
\hline & & & & & & & Meso & Braqui & Hiper \\
\hline & prom & DS & prom & DS & prom & DS & $\%$ & $\%$ & $\%$ \\
\hline Deformado & $158,48^{1}$ & 10,71 & $145,93^{2}$ & 7,58 & $92,63^{3}$ & 9,72 & $\begin{array}{ll}----- \\
---\end{array}$ & 22,2 & 77,8 \\
\hline No deformado & $167,10^{1}$ & 6,67 & $142,28^{2}$ & 6,62 & $85,25^{3}$ & 4,77 & 12,0 & 42,0 & 46,0 \\
\hline
\end{tabular}

Tabla III. Frecuencia de huesos lambdoideos según índice craneano y porcentaje de braquicefalia (n=77). 1 p<0,05=0,00000249247 con diferencia estadística significativa al $95 \%$ entre promedios de número de huesos lambdoideos entre cráneos deformados y no deformados. 2 p $<0,05=3,67073 \mathrm{E}-7$ con diferencia estadística significativa al 95\% entre porcentajes de cráneos deformados y no deformados que presentan huesos lambdoideos.

\begin{tabular}{|c|c|c|c|c|c|c|}
\hline Tipo de cráneo & Índice craneano & $\begin{array}{c}\mathrm{n}^{\circ} \text { huesos } \\
\text { lambdoideos }\end{array}$ & Der & Izq & Rango & $\begin{array}{c}\text { \% cráneos con } \\
\text { huesos } \\
\text { lambdoideos }\end{array}$ \\
\hline Deformado & 92,63 DS 9,72 & $4,67^{1} \mathrm{DS} 4,22$ & 2,4 & 2,3 & $0-18$ & $92,6 \%$ \\
\hline No deformado & 85,25 DS 4,77 & $1,02^{1} \mathrm{DS} 2,05$ & 0,5 & 0,5 & $0-7$ & $32,0 \%^{2}$ \\
\hline
\end{tabular}




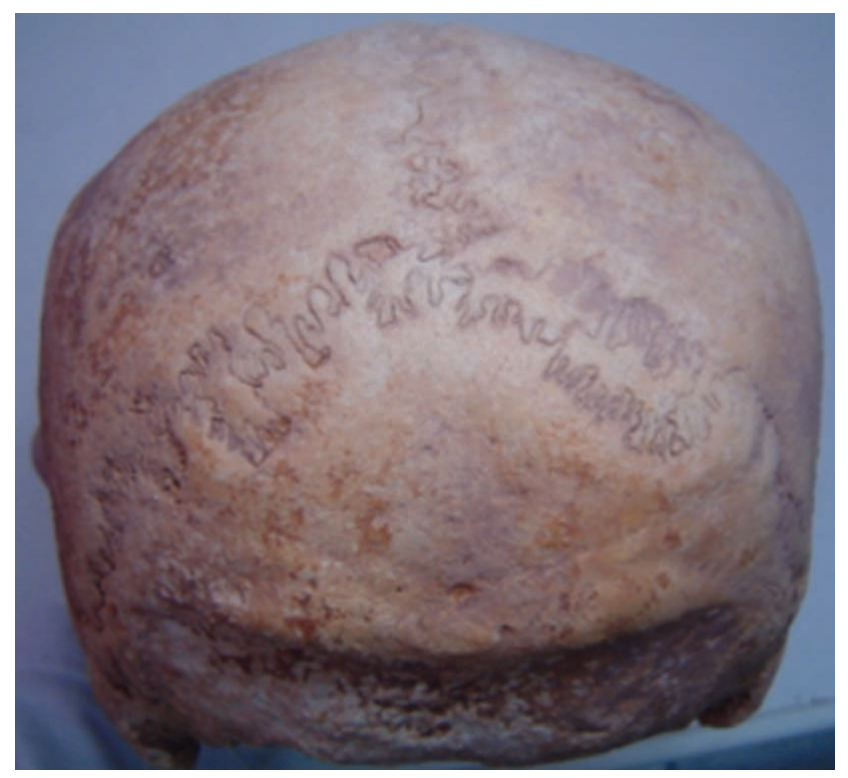

Fig. 2. Huesos lambdoideos bilaterales en cráneo deformado artificialmente.

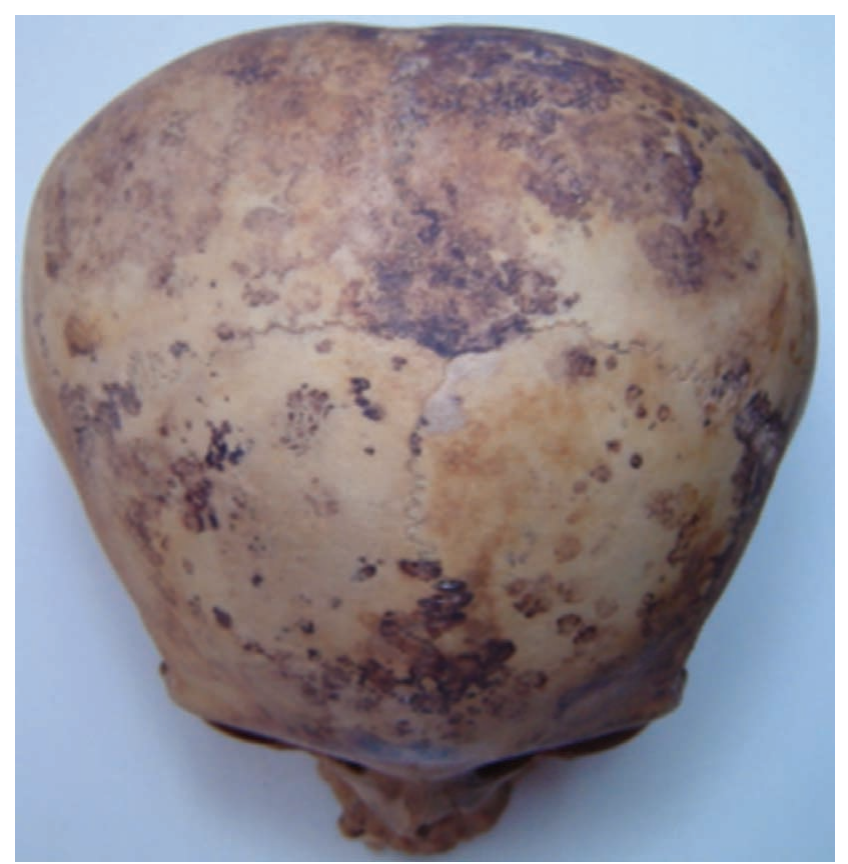

Fig. 3. Visión en norma superior de cráneo deformado artificialmente de forma braquicéfala.

\section{DISCUSIÓN}

Es indudable que la deformación craneal artificial afecta el normal crecimiento de los huesos anchos del cráneo dada su gran maleabilidad en edades tempranas, alterando la disposición de la suturas y estimulando la formación de centros secundarios de calcificación que originan huesos wormianos, en especial a nivel de los fontículos laterales y de las suturas lambdoideas.

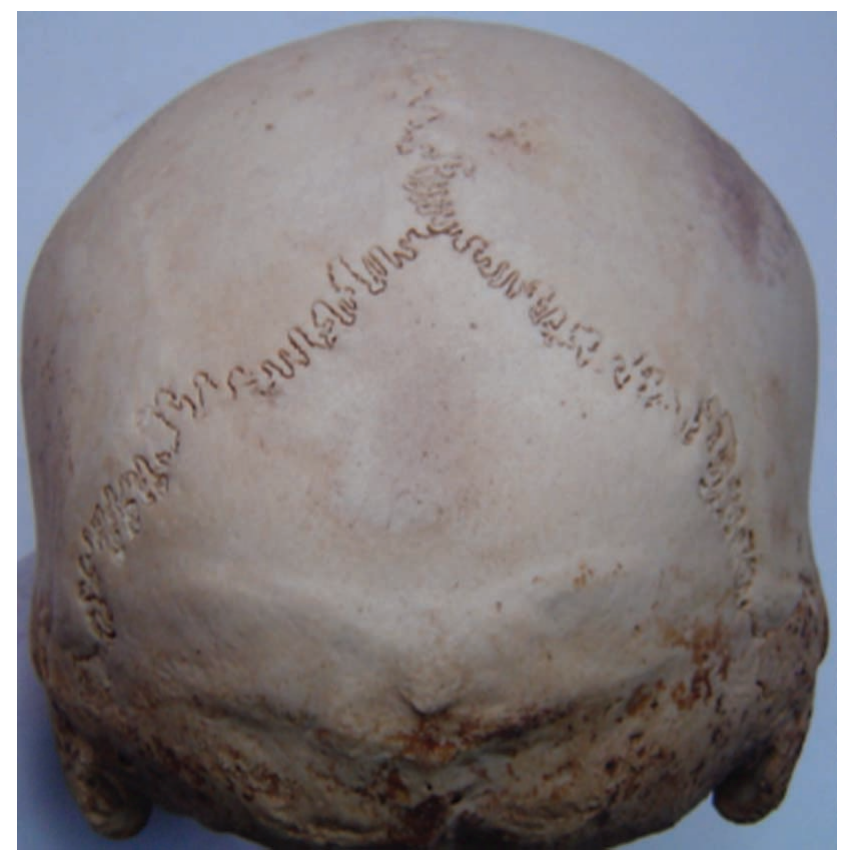

Fig. 4. Ausencia de huesos lambdoideos Visión cráneo no deformado.

El análisis muestra diferencias estadísticas significativas al 95\% al comparar longitud anteroposterior máxima del cráneo entre atacameños con bóveda craneal no deformada y con deformación artificial $(\mathrm{p}<0,05=0,0000424296)$ y anchura máxima del cráneo en atacameños con y sin deformación craneal artificial ( $\mathrm{p}<0,05=0,0313775)$ (Tabla II.).

Lo mismo se comprueba al comparar los índices craneanos de atacameños con deformación craneal (Tabla II), los cuales presentan una mayor hiperbraquicefalia que los cráneos no deformados $(77,8 \%-46 \%)$, con una diferencia estadística significativa al $95 \%(\mathrm{p}<0,05=0,0000266496)$. La mayor braquicefalia en cráneos deformados confirma lo señalado por Sánchez-Lara et al.; Carod-Artal \& VásquezCabrera; Del Papa \& Pérez.

El promedio de 4,67 (DS 4,22) huesos lambdoideos en cráneos deformados muestra una diferencia estadística significativa al $95 \%(\mathrm{p}<0,05=0,00000249247)$ al compararse con cráneos no deformados (Tabla III.), lo cual confirma lo señalado por O'Loughlin y Carod-Artal \& Vásquez-Cabrera, en relación a que la deformación craneal determina el desarrollo de huesos wormianos en las suturas de la parte posterior del cráneo. En contraposición a nuestros resultados, Sánchez-Lara et al. analizaron índices craneanos de tipo braquicefálico con deformación craneal, no observando correlación estadística significativa con el aumento del número de huesos wormianos. 
Sin embargo, los mismos autores Sánchez-Lara et al. señalan que existe un aumento del número de huesos wormianos a medida que se incrementa la braquicefalia, antecedente que fue confirmado en nuestra investigación, donde el 92,6\% de los cráneos deformados presentan huesos lambdoideos y a su vez muestran una mayor braquicefalia ( $<<0,05=3,67073 \mathrm{E}-7$ con diferencia estadística significativa al 95\%) si se comparan con cráneos no deformados, donde solamente se determina un $32,0 \%$ de los cráneos tienen huesos lambdoideos (Tabla III).

\section{CONCLUSIONES}

Los cráneos atacameños son braquicefálicos, existiendo mayor hiperbraquicefalia en los cráneos con deformación craneal ( $78 \%-46 \%$ con $p<0,05$ significativo). El tipo de deformación tabular provoca braquicefalia o la incrementa (Fig. 3.), confirmando lo señalado por la literatura respecto del método de deformación tabular (Torres-Rouff). La frecuencia de huesos lambdoideos predomina en cráneos atacameños braquicéfalos ( $\mathrm{p}<0,05$ de significancia). En los cráneos deformados se altera el cierre sutural, estimulando la formación de huesos suturales o wormianos (Carod-Artal \& Vásquez-Cabrera), especialmente en suturas posteriores (O'Loughlin). La deformación craneal aumenta el porcentaje de cráneos braquicéfalos que presentan huesos lambdoideos (Del Papa \& Pérez).

\section{AGRADECIMIENTOS}

Los autores agradecen al Prof. Dr. Mark Hubbe, Director del Instituto de Investigaciones Arqueológicas y $\mathrm{Mu}-$ seo R.P. Gustavo Le Paige en San Pedro de Atacama dependiente de la Universidad Católica del Norte, Chile, por su aporte y colaboración para la revisión del material antropológico atacameño utilizado en la presente investigación.

GARCÍA-HERNÁNDEZ, F. \& MURPHY-ECHEVERRÍA, G. Frecuency of wormian lambdoid bone in skulls with artificial deformation in Northern Chile. Int. J. Morphol., 27(3):933-938, 2009.

SUMMARY: Sutural or wormians bones are accesory bones, with genetic and heriditary importance, and consided ethnic variables (Orts Llorca, 1958; Figún \& Garino, 1992). They are characterized by be variables in number, size and shape, located in sutures and fontanelles, preferably in posteriorly placed sutures (Sappey 1888, Sicher, 1965; Sánchez-Lara et al., 2007). They are of interest in human anatomy, physical anthropology, imagenology and legal medicine (Lips \& García-Hernández, 1983; Henríquez-Pino et al., 1992; Braga et al., 2000; García-Hernández et al., 2007). Cranial deformation, pathological or artificial, stimulates the formation of accidental ossification centers, increasing the formation of ossicles in sutures and fontanelles (Del Papa \& Pérez, 2007). Artificial cranial deformation was common in amerindian cultures, for aesthetic, magical or religious purpose, as a form of ethnic identificate, social, nobility or associated with the ruling group (Torres-Rouff, 2007) and it was performed applying wooden boards or bandages in the head of newborn. The hypothesis of investigation is to determinate if there is a greater number of ossicles lambdoid in skulls of atacameños in Northern Chile with and without artificial cranial deformation. To fulfill this goal we studied 77 atacameños skulls of both sexes ( 27 deformed y 50 not deformed), selected at random between 293 individuals of the Coyo-Orient cementery, dated between 300-1200 A.C. and these remains are part of the Museum R.P. Gustavo Le Paige in San Pedro de Atacama of the North Catholic University. All skulls were analyzed, photographed in all anatomical norms and measured to obtain the cranial index. There prevalence of lambdoid bones in artificial cranial deformed ( $\mathrm{p}<0,05=0,00000249247)$, with average cranial index with greater hyperbrachycephaly (92,63 DS 9,72) and greater percentage of brachycephalic skulls with lambdoid bones $(92,6 \%-32 \%$; $p<0,05=3,67073 \mathrm{E}-7)$.

KEY WORDS: Brachycephaly; Lambdoid bone; Artificial cranial deformation; Northern Chile.

\section{REFERENCIAS BIBLIOGRÁFICAS}

Braga, M. T. T.; Gabrielli, C.; De Souza, A.; Rodrigues, C. F. S. \& Marino, J. C. Sutural bones in the pterion. Rev. Chil. Anat., 18(1):7-101, 2000.

Carod-Artal, F. J. \& Vásquez-Cabrera, C. B. Neurological paleopathology in the pre-columbian cultures of the coast and the andean plateau (I) artificial cranial deformation. Rev. Neurol., 38(8):791-7, 2004.
Del Papa, M. C. \& Pérez, I. The influence of artificial cranial vault deformation on the expression of cranial nonmetric traits: its importance in the study of evolutionary relationships. Am. J. Physical Anthropol., 134:251-62, 2007.

El-Najjar, M. \& Dawson, G. L. The effect of artificial cranial deformation on the incidence of wormian bonesin the lambdoid sutura. Am. J. Phys. Anthropol. 46(1):155-60, 1977. 
Figún, M. E. \& Garino, R. R. Anatomía Odontológica funcional y aplicada. $2^{\mathrm{a}}$ ed. Buenos Aires, El Ateneo, 1992.

García-Hernández, F.; Díaz, B. J. L. \& Murphy, E. G. T. Incidencia de huesos suturales en cráneos de changos. Int. J. Morphol., 25(4):801-4, 2007.

Henríquez-Pino, J.; Buarque, L. C. \& Prates, J. C. Anatomical study of sutural bones in 200 Brazilian individuals skulls. Rev. Chil. Anat., 10(2):89-95, 1992.

Kahle, W.; Leonhardt, H. \& Platzer, W. Atlas de Anatomía para estudiantes y médicos. $1^{\mathrm{a}}$ ed. Barcelona, Omega, 1977.

Lips, W. \& García-Hernández, F. Estudios comparativos de la presencia de huesos wormianos en cráneos indígenas y su comparación con otras muestras poblacionales de Chile. Anal. Anat. Normal, 1:95-9, 1983.

Meskell, L. The irresistible body and the seductions of achaeology. Changing bodies, changing meanings: Studies on the human body in antiquity. Montserrat, D. (Ed). New York, Routledge, 1998. pp.139-61.

O'Loughlin, V. D. Effects of different kinds of cranial deformation on the incidence of wormian bones. Am. J. Physical Anthrop., 123(2):146-55, 2004.

Orts-Llorca, F. Anatomía Humana. $2^{\mathrm{a}}$ ed. Barcelona, Editorial Científico Médica, 1958.

Ossenberg, N. S. The influence of artificial cranial deformation of wormian bones. Am. J. Phys. Anthrop., 33:375-82, 1970.

Sappey, PH. C. Traité d'Anatomie Descriptive. $4^{\mathrm{a}}$ ed. París, Adrien Delahaye et Emile Lecroisner Editeurs, 1888.

Sánchez-Lara, P. A.; Graham Jr., J. M.; Hing, A. V.; Lee, J. \& Cunningham, M.. The morphogenesis of wormian bones: A study of craniosynostosis and purposeful craneal deformation. Am. J. Phys. Anthropol., 123(2):146$55,2004$.

Shijman, E. Artificial craneal deformation in the precolumbian Andes. Childs Ner. Syst., 21:939, 2005.

Sicher. Oral Anatomy. $4^{\mathrm{a}}$ ed. Saint Louis, The C.V. Mosby Company, 1965.

Testut, L. \& Latarjet, A. Tratado de Anatomía Humana. 9a ed. Barcelona, Salvat, 1959.
Torres-Rouff, C. La deformación craneana en San Pedro de Atacama. Estudios Atacameños: Arqueología y Antropología Surandinas, 33:25-38, 2007.

Dirección para correspondencia:

Fernando García-Hernández

Unidad de Anatomía, Departamento Biomédico

Facultad de Ciencias de la Salud

Universidad de Antofagasta, Antofagasta, CHILE

Coquimbo 945 Depto. 64

Antofagasta - CHILE

Email: fgarcia@uantof.cl

Recibido : 27-02-2009

Aceptado: 18-05-2009 